\title{
Tratamento Psíquico Prévio ao Ingresso na Universidade: Experiência de um Serviço de Apoio ao Estudante
}

\author{
Mental Health Treatment prior to University Admission: \\ Experience of a Student Support Service
}

\author{
Andreia Maria Camargos Rocha ${ }^{I}[$ [D \\ Maria Bernadete de Carvalho ${ }^{I}(\mathbb{D}$ \\ Cristina Petersen Cypriano ${ }^{I}(\mathbb{D}$ \\ Maria Mônica Freitas Ribeiro ${ }^{I}$ (ID
}

\section{PALAVRAS-CHAVE}

- Educação de Graduação em Medicina.

- Estresse Psicológico-

- Estudantes de Medicina.

- Tratamento Farmacológico.

- Psicoterapia.

Introdução: Dados da literatura apontam para um aumento da frequência de sofrimento mental entre estudantes de Medicina. Entretanto, os fatores responsáveis ou que têm contribuído para isso ainda não estão completamente esclarecidos. Conhecê-los é fundamental para o planejamento de um serviço de apoio que acolha, identifique, acompanhe e, se necessário, encaminhe o estudante com sofrimento mental. Método: Avaliamos, neste trabalho, o relato de tratamento psíquico, anterior ao ingresso na universidade, de estudantes do curso de Medicina da Universidade Federal de Minas Gerais que buscaram atendimento no Núcleo de Apoio Psicopedagógico aos Estudantes da Faculdade de Medicina (Napem). Os relatos, os dados sociodemográficos e os motivos que levaram o aluno a procurar o serviço foram obtidos de uma ficha de inscrição preenchida pelo estudante. Resultados: No ano avaliado, 87 estudantes (47 homens, média de idade 23,3 anos) procuraram os serviços do Napem. Desses alunos, 42 (48,3\%) cursavam os dois primeiros anos; 24 (27,6\%), o terceiro e quarto anos; e 21 (24,1\%), o quinto e sexto anos. Tratamento psíquico antes do ingresso na universidade foi relatado por 53 (60,9\%) alunos: 37 (42,5\%) declararam ter feito uso de medicamentos psicoativos e 16 relataram tratamento com psicoterapia sem medicação. Os relatos de tratamento psíquico e de uso de medicamentos psicoativos antes do ingresso na universidade foram significativamente mais frequentes ( $p=0,04$ e $p=0,04$, respectivamente) entre os estudantes que cursavam os dois primeiros anos do curso do que entre os dos demais anos. Não se observaram diferenças estatisticamente significativas em relação à média de idade $(p=0,06)$, à distribuição por sexo $(p=0,87)$, à procedência $(p=0,68)$ ou ao tipo de moradia em Belo Horizonte $(p=0,96)$ quando se compararam os grupos com e sem relato de tratamento psíquico. Dentre os motivos apontados para a busca de atendimento no Napem, "ansiedade", "depressão/sintomas depressivos" $e$ "instabilidade de humor" foram relatados por 59 (67,8\%) estudantes. Problemas relacionados ao curso foram declarados por cinco (5,7\%) alunos. Conclusão: Os resultados do estudo sugerem que o tratamento psíquico prévio ao ingresso na universidade pode ser um dos fatores que contribuem para o sofrimento mental do estudante de Medicina durante o curso. 


\section{KEYWORDS}

- Undergraduate Medical Education.

- Psychological Distress.

- Medical Students.

- Drug Therapy.

- Psychotherapy.

\section{ABSTRACT}

Introduction: Data published in literature point to an increased frequency of psychological distress among medical students. However, the causes or potentially contributory factors for this scenario are not completely understood. Understanding these factors is essential for planning a support service that welcomes, identifies, monitors and, if necessary, guides the student with mental suffering. Methods: We evaluated the pre-university admission mental health treatment report on medical students at the Federal University of Minas Gerais who sought care at the Psychopedagogical Support Center. These data, as well as demographic data and the reasons that led the student to seek the service were obtained from an enrollment form filled out by the student. Results: A total of 87 students ( 47 males, mean age 23.3 years) were received support from the NAPEM in the year under study. Of those, 42 (48.3\%) were in the first two years of the course, $24(27.6 \%)$ in the $3^{\text {rd }} / 4^{\text {th }}$ year and 21 (24.1\%) in the $5^{\text {th }} / 6^{\text {th }}$ year. Mental Health treatment prior to university admission was reported by 53 (60.9\%) students: 37 (42.5\%) reported the use of psychoactive drugs and 16 reported psychotherapies without the use of medication. Mental Health treatment and medication prior to university admission were significantly more frequent ( $p=0.04$ and $p=0,04$, respectively) among students in the first two years of the course than among those in other years. No statistically significant differences in relation to the average age $(p=0.06)$, distribution by sex $(p=0.87)$, origin ( $p=0.68)$ or type of housing in Belo Horizonte $(p=0.96)$ were found when comparing those who had undergone psychic treatment with those who had not. Anxiety, depression/depressive symptoms and mood swings were reported by 59 (67.8\%) students as reasons to seek help. Problems related to the course were reported by five (5.7\%) students. Conclusion: The results of the present study suggest that Mental Health treatment prior to university admission may be one of the factors that contribute to the medical student's mental suffering during the course.

Recebido em 5/3/20

Aceito em 16/4/20

\section{INTRODUÇÃO}

$\mathrm{O}$ debate público e midiático em torno do sofrimento e do adoecimento mental entre universitários tem sido reaberto com frequência nos últimos anos, ocasionado por eventos trágicos ou pela sensibilidade da sociedade às queixas e demandas crescentes endereçadas aos profissionais da saúde mental e a este campo do saber de uma forma geral $^{1-4}$. Com nossa pesquisa visamos contribuir para esse debate, trazendo algumas reflexões a respeito de dados colhidos em um serviço de apoio psicopedagógico voltado para estudantes da Faculdade de Medicina da Universidade Federal de Minas Gerais (UFMG), onde são ministrados os cursos de Medicina, Fonoaudiologia e Tecnologia em Radiologia.

O Núcleo de Apoio Psicopedagógico aos Estudantes da Faculdade de Medicina (Napem) foi criado em 2004 como um órgão de assessoramento da diretoria para assuntos relativos a questões de ordem pedagógica e psicossocial que afetam individual ou coletivamente os estudantes da Faculdade de Medicina da $\mathrm{UFMG}^{5}$. A necessidade de apoio psíquico-pedagógico ao estudante de Medicina que levou a criação do Napem se repete na construção de serviços semelhantes em outras faculdades brasileiras ${ }^{6,7}$.

Hoje as iniciativas se multiplicam e são muitas as ações para manter serviços desse gênero. A preocupação com a saúde mental dos estudantes tem sido crescente, com vários trabalhos publicados ${ }^{8}$ e grupos de trabalho ativos, como o Fórum Nacional de Serviços de Apoio ao Estudante de Medicina (Forsa) da Associação Brasileira de Educação Médica (Abem) que reúne pessoas de todos os serviços de apoio aos estudantes.

Apesar do empenho de várias faculdades em oferecer suporte psíquico a seus estudantes, questões sobre o melhor tipo de abordagem, acompanhamento e encaminhamento dos alunos permanecem em debate.
Uma das possíveis razões para isso é que os fatores responsáveis ou que têm contribuído para o adoecimento mental dos estudantes ainda não estão completamente esclarecidos ${ }^{9-11}$.

Neste trabalho, interessamo-nos por analisar, com base nos dados contidos na ficha preenchida pelos alunos no momento em que realizam a demanda de atendimento ao Napem, a ocorrência de tratamento psicológico e/ou psiquiátrico antes do ingresso na universidade. Nossa hipótese é que o sofrimento mental anterior ao ingresso na universidade é um dos fatores que podem levar ao aumento da fragilidade psíquica do aluno, tornando-o mais suscetível às pressões do curso médico.

Considerando ainda que as condições sociais do estudante constituem variável cuja relevância já foi indicada por estudos realizados em serviços similares ao Napem ${ }^{12-14}$ e que diversas mudanças foram introduzidas nas formas de ingresso nas universidades federais com o Sistema de Seleção Unificada (Sisu) e a Lei de $\operatorname{Cotas}^{15}$, como maior mobilidade geográfica dos alunos e, consequentemente, maior distância do convívio familiar, afastamento de núcleos de sociabilidade já estabelecidos e incipiência dos novos relacionamentos, nosso levantamento levou em conta também as circunstâncias sociodemográficas desses estudantes.

\section{METODOLOGIA}

O Napem acolhe estudantes de graduação dos cursos de Medicina, Fonoaudiologia e Tecnologia em Radiologia, ofertados pela Faculdade de Medicina da UFMG, que o procuram por demanda espontânea. Este trabalho foi desenvolvido com base nas informações obtidas da ficha de inscrição preenchida pelo próprio aluno ao buscar, pela primeira vez, o atendimento no Napem, na qual, além de dados sociodemográficos, como curso, período, idade, sexo, procedência e tipo de moradia em Belo 
Horizonte, constavam as seguintes perguntas:

1. Você já fez tratamento psicológico ou psiquiátrico anteriormente ao seu ingresso no curso de graduação?

( ) Não

( ) Sim, psicoterapia

( ) Sim, uso de medicamentos psicoativo

( ) Sim, psicoterapia e medicamentos psicoativos

2. Se você respondeu afirmativamente:

Se medicamento:

1 - Qual(is)?

2 - Por quanto tempo?

Se terapia: Por quanto tempo?

\section{Por que você está procurando o Napem?}

Analisaram-se os dados de todos os alunos do curso de Medicina que fizeram sua inscrição no Napem durante um ano-calendário, no período compreendido entre 2016 e 2018. O ano não está explicitado para evitar qualquer possibilidade de identificação dos alunos. $\mathrm{O}$ curso tem a duração de seis anos e recebe anualmente 320 alunos. No ano avaliado, estavam matriculados 1.986 estudantes (1.066 homens e 920 mulheres).

Tabularam-se as variáveis para cálculo de frequência, e as comparações foram feitas empregando o teste do qui-quadrado. Para comparação entre médias, empregou-se o teste t de Student. Na análise dos dados, adotou-se o programa estatístico SPSS versão 20.0.

O projeto foi aprovado no Comitê de Ética e Pesquisa da UFMG (Certificado de Apresentação para Apreciação Ética (CAAE) no 73541817.2.0000. 5149).

\section{RESULTADOS}

Incluíram-se no estudo 87 alunos (47 do sexo masculino e 40 do sexo feminino). A média de idade foi de $23,3( \pm 3,7)$ anos, e a faixa etária variou de 18 a 38 anos. Vinte e seis alunos (29,9\%) eram de Belo Horizonte, cidade na qual se localiza a faculdade, e $61(70,1 \%)$ provinham de outras cidades tanto do estado de Minas Gerais quanto de outros estados (São Paulo, Espírito Santo, Bahia, Paraná e Mato Grosso do Sul). Quarenta estudantes $(46,0 \%)$ residiam com os pais ou com parentes, $27(31,0 \%)$ residiam em repúblicas estudantis ou com amigos e 20 (23,0\%) moravam sozinhos em Belo Horizonte.

Com relação à sua localização temporal no curso, 42 (48,3\%) estudantes estavam nos dois primeiros anos, 24 (27,6\%) no terceiro ou quarto ano e $21(24,1 \%)$ nos dois últimos anos. Considerando uma média de 320 alunos/ano inscritos na Faculdade de Medicina, a procura pelo Napem foi significativamente maior $(p=0,009)$ entre os que estavam nos dois primeiros anos do curso.

Tratamento psíquico anterior ao ingresso na universidade foi relatado por $53(60,9 \%)$ alunos, dos quais 37 (42,5\% do total) declararam ter feito uso de medicamentos psicoativos (associado ou não à psicoterapia) e 16 fizeram psicoterapia sem o uso de medicamentos. Entre os medicamentos utilizados pelos estudantes, foram citados antidepressivos da classe dos inibidores da recaptação da serotonina (ou serotonina/noradrenalina ou dopamina/noradrenalina/serotonina), antidepressivos da classe dos tricíclicos/tetracíclicos e ansiolíticos, como os benzodiazepínicos, estabilizadores de humor, anti-psicóticos, metilfenidato, indutores do sono e prometazina. Os períodos de tratamento medicamentoso e psicoterápico variaram de um mês a cinco anos e de dois meses a dez anos, respectivamente.

O relato de tratamento psíquico antes da entrada na universidade foi significativamente mais frequente $(p=0,04)$ entre os estudantes que cursavam os dois primeiros anos do que entre os que cursavam os demais anos (Tabela 1). O relato do uso de medicamentos também foi mais frequente $(\mathrm{p}=0,046)$ entre os alunos dos dois primeiros anos $(21 / 42,50 \%)$ quando comparados aos dos dois últimos anos do curso (5/21, 23,8\%).

Entretanto, não se observaram diferenças estatisticamente significativas na média de idade, na distribuição por sexo, na procedência ou no tipo de

\begin{tabular}{|c|c|c|c|c|}
\hline \multicolumn{5}{|c|}{$\begin{array}{ll} & \text { Tabela } 1\end{array}$} \\
\hline & \multicolumn{4}{|c|}{ Tratamento psíquico prévio } \\
\hline & & Sim & Não & Valor de $p$ \\
\hline Idade (anos) & Média (DP) & $22,7(3,3)$ & $24,2(4,3)$ & 0,06 \\
\hline \multirow{2}{*}{ Sexo } & Masculino (\%) & $29(33,3)$ & $18(20,7)$ & \\
\hline & Feminino (\%) & $24(27,6)$ & $16(18,4)$ & 0,87 \\
\hline \multirow{3}{*}{ Ano cursado } & $1^{\circ}$ e $2^{\circ}(\%)$ & $30(34,5)$ & $12(13,8)$ & \\
\hline & $3^{\circ}$ e $4^{\circ}(\%)$ & $15(17,2)$ & $9(10,3)$ & \\
\hline & $5^{\circ}$ e $6^{\circ}(\%)$ & $8(9,3)$ & $13(14,9)$ & 0,04 \\
\hline \multirow{2}{*}{ Procedência } & Belo Horizonte (\%) & $15(17,2)$ & $11(12,6)$ & \\
\hline & Outras cidades $(\%)$ & $38(43,8)$ & $23(26,4)$ & 0,68 \\
\hline \multirow{3}{*}{ Tipo de moradia em Belo Horizonte } & Pais/parentes (\%) & $24(27,6)$ & $16(18,4)$ & \\
\hline & Sozinho (\%) & $12(13,7)$ & $8(9,3)$ & \\
\hline & República/amigos (\%) & $17(19,5)$ & $10(11,5)$ & 0,96 \\
\hline
\end{tabular}

Fonte: Elaborada pelas autoras.

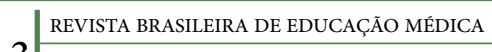

3 44 (3) : e077; 2020 
moradia em Belo Horizonte quando se compararam os grupos de estudantes com e sem relato de tratamento psíquico prévio (Tabela 1).

Dentre os motivos para a busca de atendimento no Napem, predominaram "ansiedade", "depressão/sintomas depressivos" e "instabilidade de humor" que foram relatados por 59 (67,8\%) estudantes. "Ideação de morte/ suicídio" ou "planejamento de autoextermínio" e/ou "tentativas prévias de autoextermínio/suicídio" estavam presentes em 12 relatos.

Questões como conflitos familiares ou internos, doença de um dos pais ou do próprio aluno, problemas financeiros, dificuldade de adaptação à cidade, dificuldade com a separação dos pais e uso de álcool e/ou drogas foram relatados por 17 (19,5\%) alunos. Questões relacionadas especificamente ao curso foram relatadas por cinco $(5,8 \%)$ alunos. Dois deles, alunos do último ano, relataram ansiedade por causa da proximidade da finalização do curso, um mencionou ansiedade provocada pelo internato rural, um queixou-se de pressão com cobranças do curso, e um afirmou que queria se desligar do curso, mas não apresentou possíveis justificativas para isso. Seis alunos (6,9\%) demandaram atendimento por outras razões.

\section{DISCUSSÃO}

Apesar de a amostra que constitui este estudo - estudantes que demandaram de forma espontânea atendimento no Napem - não representar, em nenhum dos parâmetros analisados, a população de estudantes da Faculdade de Medicina, algumas breves considerações merecem ser feitas a partir da comparação com os dados apresentados por Nonato ${ }^{16}$ em sua pesquisa sobre os processos de escolha dos cursos superiores e o perfil dos estudantes que ingressaram na UFMG em 2012 e 2016. A autora utiliza dados gerados pela Pró-Reitoria de Graduação (Prograd) da UFMG e por um questionário próprio aplicado aos alunos.

No que diz respeito à idade e ao sexo dos ingressantes no curso de Medicina, Nonato ${ }^{16}$ observa o predomínio daqueles com idades compreendidas entre 18 e 24 anos, bem como de estudantes do sexo masculino. Os dados da nossa amostra - média de idade de 23,3 anos e $54 \%$ estudantes do sexo masculino - parecem semelhantes aos da autora, não sugerindo que haja uma concentração da demanda por atendimento no Napem em função da idade ou do sexo.

No que se refere à procedência e ao tipo de moradia atual, variáveis provavelmente relacionadas entre si, a maioria dos estudantes $(70,1 \%)$ que procurou o Napem provinha de outras cidades que não Belo Horizonte, onde se localiza a Faculdade de Medicina da UFMG. Ainda, e talvez em consequência disso, a maioria (54\%) morava, em Belo Horizonte, em repúblicas estudantis, com amigos ou sozinhos. A maior porcentagem de demandas dirigidas ao Napem por parte de alunos provenientes de outras cidades que não Belo Horizonte tampouco evidencia uma característica particular dessa clientela, já que, por causa das possibilidades abertas pelo Sisu, tem-se hoje na UFMG e, em particular, no curso de Medicina um maior número de estudantes provenientes do interior do estado e de outros estados, com relação aos procedentes de Belo Horizonte e região metropolitana. Conforme assinala Nonato ${ }^{16}$, após a implantação do Sisu, "a concorrência por esse curso [...] deixou de ser predominantemente local para ser nacional (p.198-99)".

A análise dos dados relativos ao ano cursado pelo estudante quando procurou o atendimento no Napem mostrou predominância dos alunos que estavam nos dois primeiros anos do curso. Estudos com estudantes de Medicina em Portugal revelaram maior estresse psicológico nos primeiros anos do curso ${ }^{17}$. É notável que os primeiros anos do curso sejam marcados por fortes mudanças na rotina escolar dos estudantes, que se deparam com uma redefinição das dinâmicas de ensinoaprendizado e com a constituição de novos núcleos de sociabilidade entre os colegas. Tais mudanças frequentemente exigem dos alunos algum tipo de reposicionamento subjetivo no que diz respeito às relações que estabelecem com as figuras de autoridade e também com os próprios pares ${ }^{18}$. Tais exigências podem acarretar sofrimento psíquico, principalmente para os estudantes de Medicina que habitualmente enfrentam intensos processos preparatórios para ingressar no curso e descobrem que o alcance da meta não constitui o fim da sobrecarga, mas sim um recomeço repleto de novos desafios

Em relação ao relato de tratamento psíquico prévio ao ingresso na universidade, mesmo considerando que trabalhamos com uma amostra de alunos que demandaram, espontaneamente, atendimento no Napem e que essa origem condiciona e localiza nossos dados, limitando o alcance de nossas conclusões, pareceu-nos significativo que tal relato tenha sido feito por $61 \%$ dos estudantes e que, dentre eles, 37 (42,5\% do grupo total) declararam ter feito uso de medicamentos psicoativos.

Embora a forma de obtenção dos dados do presente estudo questões pontuais contidas na ficha de inscrição no Napem - não nos autorize a estabelecer diagnósticos ou avaliar os critérios empregados para a prescrição de medicamentos, podemos levantar a hipótese de que o relato de tratamento psíquico é um indício da existência de sofrimento mental. Apesar da constatação de que medicamentos psicoativos têm sido, cada vez mais, prescritos com diferentes objetivos, nas mais variadas especialidades médicas ${ }^{19,20}$, aqui, no entanto, a sua utilização parece estar associada à presença de dificuldades ou queixas de ordem psíquica, que também foram apontadas pela maior parte dos estudantes (68\%) como justificativa para a busca de apoio no Napem.

Partindo dessa premissa, constatamos que a frequência de sofrimento mental (61\%) prévio ao ingresso na universidade na amostra por nós analisada foi superior às taxas de transtornos depressivos $(5,8 \%)$ e de ansiedade $(9,3 \%)$ estimadas pela Organização Mundial da Saúde para a população geral do Brasil ${ }^{21}$, bem como superior às taxas descritas para o "transtorno mental comum" na nossa população (variação entre $17 \%$ e $35 \%)^{22}$. Também elevado foi o percentual de alunos que relataram uso de medicamentos psicoativos, resultados que nos parecem compatíveis com pesquisas que indicam o alto consumo de psicotrópicos na atualidade ${ }^{19,20}$.

Relato de tratamento psíquico prévio $(\mathrm{p}=0,04)$ e de uso de medicamentos $(p=0,04)$ foi visto mais frequentemente entre estudantes que cursavam o primeiro ou o segundo ano do que entre os dos demais anos. É possível que a existência de sofrimento mental antes do ingresso na universidade seja um fator predisponente ao sofrimento mental do estudante universitário, o que faz com que a busca de suporte psíquico se dê precocemente durante o curso. Também apontando para a presença de transtorno psíquico logo no início do curso médico, Puthran, Zhang, Tam e $\mathrm{Ho}^{23}$, em meta-análise que incluiu 64 estudos, observaram maior prevalência de depressão entre aqueles do primeiro ano $(33,5 \%)$ do que entre os do quinto ano (20,5\%). Embora o estudo não analise as condições de saúde mental dos estudantes antes do ingresso na universidade, é razoável supor que pelo menos uma parte desses alunos do primeiro ano tenha ingressado na universidade com algum grau de sofrimento mental.

REVISTA BRASILEIRA DE EDUCAÇÃO MÉDICA

4 44(3) : e077; 2020 
Dentre os estudos consultados, uma comunicação de pesquisa publicada em $2008^{24}$ nos chamou a atenção por trabalhar dados colhidos em questionários de pré-admissão à universidade, que interrogam, como as fichas aqui utilizadas, a respeito da existência de problemas psíquicos anteriores ao ingresso na faculdade. Trata-se de uma pesquisa retrospectiva que dispôs de informações sobre os estudantes de Medicina a partir do banco de dados do Serviço de Saúde Ocupacional de Nottingham, no Reino Unido. Constata-se, nessa pesquisa, maior frequência de relato de adoecimento mental prévio ao ingresso na faculdade entre os estudantes que se desligaram do curso quando comparados aos que o finalizaram. No curso de Medicina da UFMG, o número de alunos que desistem do curso tem sido bastante inexpressivo. De acordo com o Departamento de Registro e Controle Acadêmico da UFMG, no ano estudado, foram nove desistências formais e três exclusões por rendimento insuficiente.

Essas pesquisas indicam, tal como a nossa, a relevância da história pregressa de sofrimento mental dos estudantes na compreensão das dificuldades por eles enfrentadas em sua formação acadêmica. Uma hipótese a ser considerada é que o tratamento psíquico prévio possa ser um indicador de maior grau de fragilidade emocional, o que pode estar associado a uma maior dificuldade do indivíduo em lidar com as exigências acadêmicas do curso.

Embora não seja objetivo deste estudo (e nem seu desenho o permitiria) esclarecer a que se deve esse índice elevado de uso de medicações psicoativas entre jovens que ingressam na universidade, parece relevante tecer alguns comentários.

Alguns estudos sugerem que o adoecimento mental não só tem se tornado mais frequente na sociedade contemporânea, como também tem atingido cada vez mais a população jovem ${ }^{8,24,25,26}$. Outros enfatizam a generalização e banalização do consumo de medicamentos psicoativos na contemporaneidade, em busca de tratamento para sofrimentos psicológicos pontuais, com aumento significativo da prescrição de medicamentos antidepressivos por médicos de todas as especialidades ${ }^{19,20}$. Outros, ainda, estudam as mudanças introduzidas no saber e na prática psiquiátricos em função da descoberta de moléculas psicoativas, discutindo não só a revitalização da psiquiatria biológica, mas também os interesses econômicos, de pesquisadores, médicos e pacientes envolvidos nessa aposta ${ }^{27-29}$. Para $\operatorname{Rose}^{30}$, os avanços nesse campo vêm acumulando recursos imagéticos e de linguagem que conduzem à afirmação de uma subjetividade que se compreende a partir do funcionamento cerebral e que busca soluções medicamentosas. A relação estabelecida entre sintomas como depressão e ansiedade e os déficits ou anomalias no funcionamento do sistema de serotonina pode ser citada como um exemplo do tipo de argumento que embasa essa subjetividade. No entanto, é ainda difícil avaliar em que medida essa maneira neurobiológica de pensar substituirá os discursos psicológicos na oferta de elementos para a compreensão das dificuldades e para a sustentação de si.

Ainda em relação a essa questão, alguns resultados obtidos com a amostra avaliada merecem destaque. Assim, por exemplo, podemos interrogar se períodos muito curtos de uso de medicação (um/dois meses), presentes nos relatos, poderiam sugerir prescrições visando atuar sobre manifestações de mal-estar pontuais. Ou se esse dado poderia apontar para a não adesão ao tratamento, questão significativa no contexto do atendimento psiquiátrico e da realidade vivida pelos profissionais que atuam no Napem. Podemos também interrogar se o uso simultâneo de drogas antipsicóticas e de estabilizadores de humor, presentes em alguns relatos, poderiam indicar com grau maior de certeza a presença de doença mental. Corroborando essa possibilidade, é interessante citar que 20 dos 87 estudantes da nossa amostra foram encaminhados para acompanhamento psiquiátrico e que, dentre eles, 13 apresentavam relatos de tratamento medicamentoso prévio ao ingresso na universidade e oito de ideação suicida ou tentativa de autoextermínio.

Por fim, chama-nos a atenção o fato de que apenas cinco alunos tenham procurado atendimento no Napem por queixas relacionadas ao curso. Problemas relacionados ao internato e, especialmente, à finalização do curso, situações vividas com relativa frequência não só pelos estudantes de Medicina e provavelmente associadas à insegurança ante novas situações, foram relatados por três estudantes. Apesar de o aluno que declarou sua vontade de se desligar do curso não ter registrado seus motivos, pode-se pensar que essa situação de incerteza quanto à escolha profissional tenha sido uma causa de sofrimento psíquico, especialmente por se tratar do curso mais concorrido de uma reconhecida instituição pública de ensino superior. A equipe no Napem tem observado que esse dilema se tornou mais frequente após a instituição do Sisu como forma de seleção para admissão às universidades, quando a nota obtida, e não o desejo de exercer determinada profissão, passa a interferir na escolha do curso.

Em somente um questionário, encontramos queixa relativa às exigências do curso. De fato, a sobrecarga de horas de estudo do curso de Medicina, a competitividade entre os próprios estudantes e a insegurança provocada pelo fato de terem que lidar com o sofrimento do outro na área da saúde são fatores favorecedores e geradores de sofrimento mental em uma parcela dos alunos e não podem ser negados ou desconsiderados em nenhuma discussão sobre a saúde mental de estudantes ${ }^{31,32}$. Na interpretação dos nossos dados, é possível supor que, enquanto o sofrimento mental prévio apresenta relevância na procura de atendimento no Napem pelos alunos do início do curso, fatores contingenciais (familiares, financeiros e outros) e os associados diretamente à sobrecarga do curso podem ter maior importância para alunos dos anos mais avançados.

A mais importante limitação do presente trabalho se refere à especificidade da amostra avaliada, constituída por estudantes que procuraram espontaneamente por um serviço de suporte disponibilizado pela faculdade, o que, portanto, não permite a generalização dos resultados obtidos para o universo de alunos do curso. Além disso, a abordagem foi retrospectiva e limitada à análise de um único ano, fornecendo, portanto, dados preliminares sobre a questão proposta. Para melhor esclarecimento do assunto, serão necessários trabalhos que avaliem longitudinalmente a qualidade de vida e a percepção sobre a saúde mental dos estudantes. Nesse sentido, alguns deles já estão em andamento na instituição e poderão permitir melhor compreensão do problema.

Em conclusão, os resultados obtidos mostraram elevada prevalência de alunos com histórico de tratamento psíquico prévio ao ingresso na universidade e buscando suporte nos primeiros anos do curso, o que sugere que esse seja um fator a ser identificado e abordado nas medidas a serem tomadas pelas universidades com o propósito de melhorar a saúde mental de seus estudantes.

\section{AGRADECIMENTOS}

As autoras agradecem a todos os membros do Napem, especialmente ao Dr. Gilmar Fidelis, coordenador técnico da equipe, e ao Dr. Marco Túlio

REVISTA BRASILEIRA DE EDUCAÇÃo MÉDICA

5 44 (3) : e077; 2020 
de Aquino, psiquiatra, que gentilmente aceitou nos dar uma entrevista, o apoio na realização desse trabalho.

\section{REFERÊNCIAS}

1. Cambricoli F, Toledo LF. Aumento dos transtornos mentais entre jovens preocupa universidades. O Estado de S. Paulo. 17 set 2017 [acesso em 5 nov. 2019]. Disponível em: https://saude.estadao.com. $\mathrm{br} /$ noticias/geral,aumento-de-transtornos-mentais-entre-jovenspreocupa-universidades, 70002003562 .

2. Lara MG. O ignorado adoecimento mental dos estudantes universitários [acesso em 5 nov. 2019]. Disponível em: https:// pensaraeducacao.com.br/pensaraeducacaoempauta/o-ignoradoadoecimento-mental-dos-estudantes-universitarios/.

3. Lopes V. Pressões potencializam casos de transtorno mental de alunos e de professores da UFMG. Estado de Minas. 28 ago 2017 [acesso em 5 nov. 2019]. Disponível em: https://www.em.com.br/app/noticia/ especiais/educacao/2017/08/28/internas_educacao,895535/pressoesaumentam-casos-de-transtorno-mental-na-ufmg.shtml.

4. UFJF Notícias. Especialistas refletem sobre adoecimento psíquico no meio universitário [acesso em 5 nov. 2019]. Disponível em: https:// www2.ufjf.br/noticias/2018/09/18/especialistas-refletem-sobreadoecimento-psiquico-no-meio-universitario/.

5. Núcleo de Apoio Psicopedagógico aos Estudantes da Faculdade de Medicina da Universidade Federal de Minas Gerais [acesso em 5 nov 2019]. Disponível em: https://medicina.ufmg.br/napem/.

6. Millan LR, Marco OLN de, Rossi E, Arruda PCV. O universo psicológico do futuro médico: vocação vicissitudes e perspectivas. São Paulo: Casa do Psicólogo; 1999.

7. Millan LR, Arruda PCV. Assistência psicológica ao estudante de Medicina: 21 anos de experiência. Rev. Assoc. Med. Bras. 2008;54(1):90-4.

8. Pacheco JP, Giacomin HT, Tam WW, Ribeiro TB, Arab C, Bezerra IM, et al. Mental health problems among medical students in Brazil: a systematic review and meta-analysis. Rev. bras. psiquiatr. 2017;39(4):369-78.

9. Moutier C, Norcross W, Jong P, Norman M, Kirby B, McGuire T, et al. The suicide prevention and depression awareness program at the University of California, San Diego School of Medicine. Acad. med. 2002;87(3):320-6.

10. Hope V, Henderson M. Medical student depression, anxiety and distress outside North America: a systematic review. Med. educ. 2014;48(10):963-79.

11. Aragão JC, Casiraghi B, Mota E, Abrahão M, Almeida T de, Baylão AC, et al. Saúde mental em estudantes de Medicina. Rev Estud Invest Psicol Educ 2017; vol extra(14):38-41.

12. Cerchiari EAN, Caetano D, Faccenda O. Utilização do serviço de saúde mental em uma universidade pública. Psicol. ciênc. prof. 2005;25(2)252-65.

13. Neves MCC, Dalgalarrondo P. Transtornos mentais auto-referidos em estudantes universitários. J Bras Psiquiat 2007;56(4):237-44.

14. Oliveira MLC, Dantas CR, Azevedo RCS, Bonzato CEM. Demographics and complaints of university students who sought help at a campus mental health service between 1987 and 2004. São Paulo med. j. 2008;126(1):58-62.
15. Brasil. Lei Federal no 12.711. Disponível em: http://www.planalto.gov.br/ ccivil_03/_ato2011-2014/2012/lei/112711.htm. [acesso em 5 nov. 2019].

16. Nonato BF. Lei de Cotas e Sisu: análise dos processos de escolha dos cursos superiores e do perfil dos estudantes da UFMG antes e após as mudanças na forma de acesso às instituições federais [tese]. Belo Horizonte: Universidade Federal de Minas Gerais; 2018.

17. Roberto A, Almeida A. A saúde mental de estudantes de Medicina - estudo exploratório na Universidade da Beira Interior. Acta med. port. 2011;24(S2): 279-86

18. Almeida LS, Soares APC, Ferreira JAG. Transição e adaptação à universidade: apresentação de um questionário de vivências acadêmicas (QVA). Psicologia 2000;14(2)189-208.

19. Pelegrini MRF. $O$ abuso de medicamentos psicotrópicos na contemporaneidade. Psicol. ciênc. prof. 2003;21(3):38-43.

20. Horwitz AV, Wakefield JC. A tristeza perdida. Como a psiquiatria transformou a depressão em moda. São Paulo: Summus; 2010.

21. World Health Organization. Depression and other common mental disorders: global health estimates. Geneva: WHO; 2017 [acesso em 27 fev. 2020]. Disponível em: https://www.who.int/mental_health/ management/depression/prevalence_global_health_estimates/en/.

22. Santos GBV, Alves MCGP, Goldbaum M, Cesar CLG, Gianini RJ. Prevalência de transtornos mentais comuns e fatores associados em moradores da área urbana de São Paulo, Brasil. Cad. saúde pública 2019;35(11):e00236318.

23. Puthran R, Zhang MWB, Tam WW, Ho RC. Prevalence of depression amongst medical students: a meta-analysis. Med. educ. 2016;50:456-68.

24. Yates J, James D, Aston I. Pre-existing mental health problems in medical students: a retrospective survey. Med. teach. 2008;30(3):319-21.

25. Cassimiro EE. Frequência do uso de psicofármacos entre jovens estudantes que cursam pré-vestibular. Adolesc. Saúde 2012;9(4)27-36 (acesso em 5 nov. 2019]. Disponível em: http://adolescenciaesaude. com/detalhe_artigo.asp?id=342.

26. Lacerda L, Ferraro M, Sena Y. Cabeça de adolescente. Folha de S.Paulo. 24 jun. 2019 [acesso em 5 nov. 2019]. Disponível em: https://www1. folha.uol.com.br/equilibrioesaude/2019/06/ansiedade-esta-entre-ostranstornos-mais-comuns-na-adolescencia.shtml.

27. Rose N. Cérebro, self e sociedade. Entrevista. Physis (Rio J.) 2010;(1):301-24.

28. Angell M. A epidemia de doença mental. Piauí; ago. 2011 [acesso em 5 nov. 2019]. Disponível em: https://piaui.folha.uol.com.br > materia > a-epidemia-de-doenca-mental.

29. Laia S. A classificação dos transtornos mentais pelo DSM-V e a orientação lacaniana. Clinicaps: Impasses da Clínica 2011;15:2 [acesso 5 nov. 2019]. Disponível em: http://www.clinicaps.com.br/clinicaps_ revista_15_art_01.html.

30. Rose N. A política da própria vida: biomedicina, poder e subjetividade no século XXI. São Paulo: Paulus; 2013.

31. Dyrbye L, Thomas MR, Shanafelt TD. Medical student distress: causes, consequences, and proposed solutions. Mayo clin. proc. 2005;80(12):1613-22.

32. MacLean L, Booza J, Balon R. The impact of medical school on student mental health. Acad. psychiatry. 2016;40:89-91. 


\section{CONTRIBUIÇÃO DAS AUTORAS}

Todas as autoras participaram da idealização do projeto, da pesquisa bibliográfica, da orientação metodológica, da análise dos dados e da redação do artigo.

\section{CONFLITO DE INTERESSES}

As autoras declaram não haver conflito de interesses neste estudo.

\section{ENDEREÇO PARA CORRESPONDÊNCIA}

Maria Mônica Freitas Ribeiro. Departamento de Clínica Médica, Faculdade de Medicina da UFMG,

Avenida Professor Alfredo Balena, 190, sala 246. Centro, Belo Horizonte, MG, Brasil. CEP: 30130-100.

E-mail: mmonica@medicina.ufmg.br 